\title{
Distinct patterns of olfactory impairment in Alzheimer's disease, semantic dementia, frontotemporal dementia, and corticobasal degeneration
}

\author{
Simona Luzzi ${ }^{\mathrm{a}, *}$, Julie S. Snowden ${ }^{\mathrm{b}}$, David Neary ${ }^{\mathrm{b}}$, Michela Coccia ${ }^{\mathrm{a}}$, \\ Leandro Provinciali ${ }^{a}$, Matthew A. Lambon Ralph ${ }^{\mathrm{c}}$ \\ ${ }^{a}$ Department of Neuroscience, University of Ancona, Italy \\ ${ }^{\mathrm{b}}$ Cerebral Function Unit, Greater Manchester Neuroscience Centre, UK \\ ${ }^{\mathrm{c}}$ School of Psychological Sciences, University of Manchester, UK
}

Received 11 April 2006; received in revised form 5 December 2006; accepted 12 December 2006

Available online 28 December 2006

\begin{abstract}
Performance on tests of odour discrimination, naming, and matching was compared in patients with four distinct forms of neurodegenerative disease: Alzheimer's disease (AD), semantic dementia (SD), frontotemporal dementia (FTD), and corticobasal degeneration (CBD). The SD patients were found to have a severe impairment of identification from olfaction despite having normal discrimination, consistent with the multimodal semantic impairment characteristic of this patient group. The AD patients' poor odour discrimination suggests that a perceptual impairment is the root of their poor odour identification. Mild impairments in odour identification observed in FTD and CBD are consistent with their generalised executive dysfunction. The findings illustrate that breakdown in olfaction can occur at a perceptual or semantic level, analogous to the distinction between apperceptive and associative forms of deficit in the visual and auditory modalities. The findings add further insights into the nature of the semantic deficit in SD by exploring a hitherto neglected modality and may have relevance in explaining the altered eating habits commonly associated with SD.
\end{abstract}

(C) 2007 Elsevier Ltd. All rights reserved.

Keywords: Neuropsychology; Smell; Odour perception; Odour semantics; Neurodegeneration; Olfactory cortex

\section{Introduction}

The purpose of the present study was to examine smell perception and recognition in four neurodegenerative disorders: frontotemporal dementia (FTD), semantic dementia (SD), Alzheimer's disease (AD), and corticobasal degeneration (CBD). There are anatomical, neuropsychological, and clinical reasons why investigation of smell in these disorders is important-yet it has never been done so before in FTD and SD.

Abbreviations: SPSB, Odour Perception and Semantic Battery; AD, Alzheimer's disease; FTD, frontotemporal dementia; SD, semantic dementia; $\mathrm{CBD}$, corticobasal degeneration

* Corresponding author at: Clinica Neurologica, Dipartimento di Neuroscienze, Università Politecnica delle Marche, Ospedali Riuniti di Ancona, Via Conca 1, Torrette di Ancona, Ancona, Italy. Tel.: +39 071 5963647; fax: +39071 887262 .

E-mail address: simonaluzzi@yahoo.it (S. Luzzi).
In the olfactory system, axons of the mitral cells of the olfactory bulb course through the olfactory tracts and then via the lateral olfactory striae terminate in the amygdala and the "primary" olfactory cortex of the uncus (Nauta \& Feirtag, 1986). Projections then pass to the "association" olfactory cortex of the parahippocampal gyrus and entorhinal area. The parahippocampal gyrus receives fibres via the cingulum from the cingulate gyrus and projects into the hippocampus. These "primary" and "associational" areas are referred to as the pyriform cortex. The amygdala receives connections from middle and inferior temporal gyri (inferior association cortex), known to be critical for semantic processing, and in turn projects into the hypothalamus. Damage to pyriform cortex would be expected to lead to impairment in odour at a sensory or perceptual (apperceptive) level, whereas damage to temporal cortical-amygdala connections would be expected to lead to impairment at a semantic (associative) level. Lesion and functional neuroimaging studies (Van Hoesen, Parvizi, \& Chu, 2000) indicate that the medial orbitofrontal cortex - which 
receives input from the piriform cortex - is also implicated in several olfactory functions, including olfactory discrimination.

In neurodegenerative disease, olfaction has been examined most extensively in AD (Doty, Shaman, \& Dann, 1984; Kesslak et al., 1988; Knupfer \& Spiegel, 1986; Koss, Weiffenbach, Haxby, \& Friedland, 1988; Larsson et al., 1999; Lehrner, Brücke, Dal-Bianco, Gatterer, \& Kryspin-Exner, 1997; McCaffrey, Duff, \& Solomon, 2000; Rezek, 1987; Royet et al., 2001; Schiffman, Clark, \& Warwick, 1990; Serby, Larson, \& Kalkstein, 1991). Studies have examined threshold, familiarity, feature discrimination, identification, and recognition (see Liberini \& Parola, 2001; Martzke, Kopala, \& Good, 1997; Mesholam, Moberg, Mahr, \& Doty, 1998 for review). Despite this extensive literature, studies have not always yielded uniform results (Liberini \& Parola, 2001; Martzke et al., 1997; Mesholam et al., 1998; Parola \& Liberini, 1999). For example, olfactory threshold has been reported to be preserved in AD by some authors (Koss et al., 1988; Serby et al., 1991) but not others (Knupfer \& Spiegel, 1986; Morgan, Nordin, \& Murphy, 1995; Rezek, 1987). Some studies have reported an olfactory perceptual impairment (Doty, Reyes, \& Gregor, 1987), whereas others have not (Koss et al., 1988; Royet et al., 2001).

In contrast to $\mathrm{AD}$, there have been no studies of olfaction in SD and FTD, two clinical syndromes of frontotemporal lobar degeneration (Neary et al., 1998). SD is a multimodal disorder of meaning, in which patients have difficulty recognising the significance of words, objects, faces, and nonverbal sounds and tastes, despite normal perception of those stimuli (Bozeat, Gregory, Lambon Ralph, \& Hodges, 2000; Coccia, Bartolini, Luzzi, Provinciali, \& Lambon Ralph, 2004; Snowden, Neary, \& Mann, 1996; Snowden, Thompson, \& Neary, 2004). It would be anticipated that there would be a parallel loss of meaning for odours but no impairment in their detection and discrimination. This would be predicted, not only on cognitive grounds based on the findings in other modalities, but also on the basis of the anatomical distributions of pathology. In SD (Snowden et al., 1996) there is severe atrophy of the amygdala and middle and inferior temporal gyri with relative sparing of the hippocampal formation (hippocampus, entorhinal cortex, and parahippocampal gyrus). Thus, impairment in odour recognition ought to occur in SD because of the underlying degradation of the meaning associated with each odour (arising from the anterior, inferiolateral atrophy of this disorder).

FTD is a disorder of behaviour, characterised by striking personality change, breakdown in social conduct and executive impairments (Gustafson, Brun, \& Risberg, 1987; Neary et al., 1998). Atrophy is most prominent in the frontal lobes, although it invariably involves the anterior temporal cortex to some degree (Neary et al., 1998; Snowden et al., 1996). One might predict, therefore, some of the deficits of SD, albeit to a lesser degree. However, in view of the effortful search involved in odour identity, performance might also potentially be compromised by patients' executive impairments. In addition, orbitofrontal atrophy might also impact directly upon smell perception.

The study of odour perception and identification has additional relevance in SD and FTD in that it might potentially shed light on some of the characteristic behavioural changes of these disorders, namely alterations in dietary habits (Bathgate, Snowden, Varma, Blackshaw, \& Neary, 2001; Bozeat, Gregory et al., 2000; Ikeda, Brown, Holland, Fukuhara, \& Hodges, 2002; Miller, Darby, Swartz, Yener, \& Mena, 1995; Snowden et al., 2001). SD patients commonly exhibit food fads and are overselective in what they eat (Bathgate et al., 2001; Snowden et al., 2001), although in the later stages of illness they may attempt to eat inanimate objects. This behaviour, recorded more frequently in SD than in FTD or AD (Bathgate et al., 2001; Snowden et al., 2001 ) is consistent with the Klüver-Bucy syndrome, which has been associated with temporal lobe pathology. Evidence for the loss of semantic knowledge of odours would lend support to the view that alterations in oral behaviour in SD are crucially linked to patients' semantic impairment. That is, patients are wary of what they eat as they no longer have olfactory cues by which to judge edibility or freshness of foodstuffs and are prone to errors in differentiating edible from inedible.

FTD patients exhibit a different pattern of eating behaviour. They commonly eat excessively and indiscriminately (Neary et al., 1998; Snowden et al., 1996), yet frequently do so only when food is present and do not actively seek it out. This raises the possibility that the behaviour is related to a general stimulusboundness and failure of inhibitory control rather than to smell or taste senses per se. The finding of preserved odour perception would lend support to the view that indiscriminate eating in FTD is not a consequence of primary olfactory deficits.

CBD provides a valuable reference disorder to $\mathrm{AD}, \mathrm{SD}$ and FTD in that it is a disorder predominantly of the basal ganglia and premotor and parietal cortex, and thus spares the temporal cortex. Olfaction might therefore be expected to be preserved. Indeed the relatively few available studies of olfaction in CBD (Müller, Reichmann, Livermore, \& Hummel, 2002; Wenning et al., 1995) found no olfactory impairment. It has been suggested (Hawkes, 2003) that preserved olfaction may provide a useful diagnostic aid in distinguishing CBD from other Parkinsonian disorders. Given the low number of studies, however, further data on olfaction in this patient group are merited.

\section{Materials and methods}

\subsection{Participants}

\subsubsection{Patients}

The patient cohort comprised 40 individuals attending a specialist neurological clinic for early onset dementia (in Ancona, Italy or Manchester, England), who had a clinical diagnosis of AD, FTD, SD, or CBD (Table 1). Diagnoses were made on the basis of clinical history, neurological and neuropsychological examination, and supported by structural and functional imaging. Routine blood screening tests excluded secondary causes of dementia. No patient had a history or risk factors for cerebrovascular disease. At the time of their examination all patients had been followed up for at least 1 year, confirming the progressive nature of their disorder. Patients fulfilled currently accepted diagnostic criteria respectively for AD (McKhann et al., 1984), SD (Neary et al., 1998), FTD (Neary et al., 1998), and CBD (Lang, Riley, \& Bergeron, 1994). Patients were in the relatively early stages of disease, to minimise confounding effects of severe cognitive impairment on olfactory performance. In particular, AD patients early in the course of their disease were selected whose deficits were predominantly of memory impairment alone. Patients with severe visual perceptual, spatial, or language deficits, which would compromise patients' ability to participate in the olfactory tests, were excluded. Lateralization of damage in these patients 
Table 1

Background neuropsychology: mean $(S D)$

\begin{tabular}{|c|c|c|c|c|}
\hline & $\mathrm{AD}$ & FTD & $\mathrm{SD}$ & $\mathrm{CBD}$ \\
\hline Number of cases & 14 & 11 & 8 & 7 \\
\hline Age & $71(8)$ & $64(7)$ & $68(6)$ & $64(7)$ \\
\hline Education & $10(4)$ & $10(2)$ & $14(3)$ & $12(3)$ \\
\hline Sex male:female & $7: 7$ & $8: 3$ & $5: 3$ & $4: 3$ \\
\hline Smokers & 0 & 3 & 0 & 2 \\
\hline Disease duration & $2(1)$ & $4(2)$ & $4(1)$ & $3(1)$ \\
\hline MMSE/30 & $24(2)$ & $24(6)$ & $21(9)$ & $28(2)$ \\
\hline \multicolumn{5}{|l|}{ Language } \\
\hline Naming/40 & $38(2)$ & $34(5)$ & $9(10)$ & $38(2)$ \\
\hline Comprehension/40 & $40(0)$ & $39(2)$ & $30(7)$ & $40(0)$ \\
\hline Category fluency (animals) & $12(4)$ & $13(6)$ & $2(2)$ & $15(8)$ \\
\hline Letter fluency ( $\mathrm{F}$ words) & $11(4)$ & $8(5)$ & $5(5)$ & $9(4)$ \\
\hline \multicolumn{5}{|l|}{ Perceptuo-spatial skills } \\
\hline \multicolumn{5}{|l|}{ VOSP } \\
\hline Shape detection test $/ 20$ & $19(1)$ & $20(1)$ & $20(0)$ & $19(1)$ \\
\hline Incomplete letters/20 & $18(2)$ & $17(3)$ & $18(1)$ & $18(3)$ \\
\hline Real object decision task/20 & $16(3)$ & $14(3)$ & $15(3)$ & $16(1)$ \\
\hline Dot counting/10 & $10(0)$ & $10(1)$ & $10(0)$ & $9(1)$ \\
\hline Position discrimination/20 & $19(1)$ & $20(1)$ & $19(1)$ & $18(1)$ \\
\hline \multicolumn{5}{|l|}{ Praxis } \\
\hline Ideomotor praxis $/ 20$ & $20(0)$ & $20(0)$ & $20(0)$ & $14(2)$ \\
\hline Constructional praxis/31 & $26(3)$ & $29(2)$ & $30(1)$ & $29(1)$ \\
\hline \multicolumn{5}{|l|}{ Memory } \\
\hline Digit span & $6(1)$ & $6(1)$ & $7(1)$ & $6(1)$ \\
\hline Hopkins test total recall/36 & $14(3)$ & $21(3)$ & - & $22(3)$ \\
\hline Hopkins test delayed recall/12 & $\mathbf{0}(\mathbf{1})$ & $5(1)$ & - & \\
\hline Rey AVLT total recall/75 & $21(3)$ & $28(1)$ & $23(10)$ & 50 \\
\hline Rey AVLT delayed recall/15 & $1(1)$ & $6(1)$ & $3(1)$ & 11 \\
\hline Delayed recall Rey Figure B/32 & $4(4)$ & $21(6)$ & $23(8)$ & 25 \\
\hline \multicolumn{5}{|l|}{ Executive function } \\
\hline Weigl's sorting test/15 & $8(1)$ & $7(2)$ & $6(3)$ & $8(1)$ \\
\hline Luria's Motor Sequences $/ 50$ & $47(3)$ & $34(8)$ & $46(4)$ & $38(7)$ \\
\hline Brixton test $/ 55$ & $25(7)$ & $28(12)$ & $18(6)$ & $18(5)$ \\
\hline
\end{tabular}

Impaired performance is shown in bold; no measure of variance is shown for the CBD group on the Rey AVLT elements because memory function was assessed using the Hopkins tests on the English cases $(N=6)$ and the Rey AVLT for the Italian patients $(N=1)$-see text for details.

groups, especially in $\mathrm{CBD}$, can be highly lateralized in the early stages. As expected, CBD were lateralized but they were almost equally divided between predominantly right (three patients) and left (four cases).

In $9 / 11$ FTD patients and 6/8 SD patients there was a history of altered eating habits. Eight of the FTD patients showed gluttony and overeating, whereas one had developed food fads. All six SD patients had food fads, without gluttony. One SD patient had attempted to eat inedible objects. In none of the AD and CBD patients were altered eating habits reported. Pharmacological history did not indicate the use of medication which could potentially interfere with olfaction. The AD and SD patients involved in the study were all non-smokers, so that impaired performance on olfactory tests cannot be ascribed to a smoking habit.

\subsubsection{Controls}

Controls were healthy volunteers, drawn from the same age range as the patient groups. Exclusion criteria included a history of central nervous system disease, head trauma, psychiatric disease or upper or lower airways disease, subjective report of loss of odour, or intake of medication that might affect cognitive function. Smoking was not considered an exclusion criterion but was recorded for future reference. The initial pilot study involved 80 individuals, 38 Italian, and 42 British, whereas the main part of the study involved a smaller group of 20 people [mean age 65 years $(S D 7)$, mean education 9 years $(S D 4)$, sex $10 \mathrm{M} / 10 \mathrm{~F}$ ], who were spouses of participating patients. The rationale for involving spouses was to control for social background and likely exposure to the odours used in the tests. Eight controls were smokers.
All participants gave informed consent to the study, which was approved by the local Ethics Committee.

\subsection{Background neuropsychology}

The 40 patients had undergone neuropsychological evaluation as part of their routine clinical assessment. Table 1 shows mean performance on tasks tapping a range of cognitive abilities. Scores were compared to the published normative data on these tasks and abnormal scores are noted in bold text. Tasks administered were common to both the Italian and British samples, except for the tests exploring verbal learning (the Hopkins test was used for the English sample and the Rey AVLT for the Italian sample).

The pattern of results on these background tests reveals the typical neuropsychological profile of each group. These are summarised briefly below. General assessment with the Mini-Mental State Examination (MMSE: Folstein, Folstein, $\&$ McHugh, 1975) revealed mild to moderate impairment in the four groups. Performance on language tasks, both naming and comprehension measured by a four-choice word-picture matching test, was profoundly impaired in the SD group but unremarkable in other groups. In the SD group, verbal fluency performance was poorer for category than letter fluency whereas other groups showed the reverse pattern. Performance was within normal limits in all groups on the perceptual and spatial tests of the VOSP battery (Warrington \& James, 1991) and on a drawing test involving copying of a simplified Rey figure (Di Nuovo, 1979). Assessment of ideomotor praxis, using a common set of gestures (Spinnler \& 
Tognoni, 1987) revealed impairment in the CBD group only. Assessment of memory revealed normal immediate memory in all groups, as measured by digit span but impaired list learning (Brandt, 1991; Caltagirone, Gainotti, Masullo, \& Miceli, 1979) and visual recall performance. Impairment was most marked in the AD group. On the Weigl's sorting test (Spinnler \& Tognoni, 1987), which is a relatively undemanding test of executive function, performance did not differ across the groups. On the Brixton test (Burgess \& Shallice, 1997), the FTD group made numerically more errors than other groups, although there was variability across the group with some FTD patients performing highly abnormally and others within the normal range. The Luria Motor Sequencing test elicited most difficulty in the FTD group. In summary, the differing neuropsychological profile demonstrated by each group was concordant with the diagnosis: memory in $\mathrm{AD}$, executive function in $\mathrm{FTD}$, language in $\mathrm{SD}$, and praxis in $\mathrm{CBD}$.

\subsection{Formation of a "smell" semantic battery}

An initial pilot investigation, involving 38 Italian and 42 British normal volunteers, was carried out with the aim of identifying a set of odours that are easily recognised by people in the general population. The pilot study was particularly important in view of the cross-cultural nature of the study (involving both Italian and British patients). We anticipated that odours that are highly familiar to people in one culture might be less well recognised in another. Indeed, our initial exploration found that there was considerable individual variation in the ability to recognise many odours. A second and important motivating factor was our desire to produce a "semantic battery" (cf. Bozeat, Lambon Ralph, Patterson, Garrard, \& Hodges, 2000). The rationale behind such batteries is that a common set of concepts are tested across a number of different semantic assessments that vary the modality of input and output. Such batteries allow the researcher to probe for the status of the underlying amodal semantic representations and for any modality-specific effects. Because of these two motivating factors, we needed to select a new set of odours (rather than use an existing olfaction test) and choosing reliably recognised odours meant that normal controls performed, as expected, close to ceiling on many of the subtests (as is typical for such experimental, neuropsychological assessments). This means that the battery is ideal for testing for patients' ability to access meaning from smell and nonsmell domains but would not be the ideal design for detecting early or subtle impairments of olfaction.

From the pilot testing, we selected 16/36 substances that were consistently recognised by a high proportion of controls in each group. The substantial crossculture differences (e.g., curry was identified by 93 percent of British subjects but only 24 percent of Italians, in contrast, pepper was identified by 96 percent of Italians but only 21 percent of British) meant that, despite our best attempts, we were unable to select exactly the same items for each group. Most items $(11 / 16)$ were common to both versions (bleach, coffee, petrol, almond, aftershave, lemon, cocoa, shoe polish, paint, vinegar, and orange). However, where a substance elicited very disparate findings a matched substitute was used (ItalianEnglish: pepper-curry; ethyl alcohol-"Dettol"; rosemary-mint; lavender-"Vic"; onion-whisky). Substances were always presented in opaque glass bottles (to prevent participants from seeing the substance) and, where necessary, replaced frequently to maintain the fragrance.

\subsection{The Odour Perception and Semantics Battery (SPSB)}

The Odour Perception and Semantics Battery (SPSB) comprised five tasks. Three were strictly related to odour as they involved an olfactory input (Odour Discrimination, Odour Naming and Odour-Picture Matching). Two constituted "control tests", as they examined semantic knowledge of the items used in the first three tasks. Each task used the same 16 stimuli. The tests were administered in the following order.

\subsubsection{Odour Discrimination task}

The task comprised 16 pairs of odours, half of which were identical and half non-identical, albeit semantically related (e.g., lemon-orange, petrol-paint, cocoa-coffee). Odours in a pair were presented consecutively, each for approximately $2 \mathrm{~s}$, with a $5 \mathrm{~s}$ interval between the two and the subject was asked to judge whether the odour pairs were the same or different. As subjects were requested to keep their eyes closed throughout the test they were pre-warned when exposure to each odour was about to occur. An interval of $20 \mathrm{~s}$ separated the presentation of one pair of odours from the next. No feedback was given. Stimuli are shown in Appendix 1.

\subsubsection{Odour Naming task}

The 16 different odours were presented consecutively and the subject was asked to name them. For unnamed items the subject was asked to describe the features of the odour (i.e., strong, sweet, bitter, etc.).

\subsubsection{Odour-Picture Matching task}

The task required the subject to select, from four coloured pictures, the one that was semantically associated with the odour (e.g., car associated with the odour of petrol; cake associated with the odour of vanilla). Each set of four pictures comprised two edible and two non-edible items, only one of the alternatives being plausible. The test was preceded by a two-item familiarisation task, using two odours not used in the main test. This familiarisation procedure was designed to ensure that the participant searched for an item semantically related to the odour (e.g., vanilla-cake) and not the source of the odour itself (e.g., vanilla-vanilla sticks). Stimuli are shown in Appendix 1.

\subsubsection{Picture Naming task}

Coloured pictures of objects/substances (e.g., almonds) corresponding to the 16 odours were presented sequentially and the subject was asked to name them.

\subsubsection{Word-Picture Matching task}

The same set of coloured pictures used in the Odour-to-Picture Matching task (task 3) were re-presented. The examiner gave a spoken word, corresponding to the odours used in tasks 1-3 (e.g., "coffee", "almond") and asked the subject to point to the picture most associated with that word.

\subsection{Analysis}

Group comparisons were examined using one-way ANOVAs. Post hoc Tukey HSD pairwise comparisons were made to identify the source of significant group effects. Correlations between experimental tests were undertaken using Spearman's $\rho$.

\section{Results}

Group means and standard deviations for each test are shown in Table 2. Performance of the four patient groups on each of the subtests is summarised below.

\subsection{Odour Discrimination}

A one-way ANOVA revealed a significant effect of group $(F(4,55)=7.9, p<.0001)$. Post hoc comparisons showed that only the AD group performed significantly worse than controls $(p<.0001)$. The AD group also performed significantly more poorly than each of the other patient groups (AD versus FTD $p=.007$; $\mathrm{AD}$ versus $\mathrm{SD} p=.003 ; \mathrm{AD}$ versus $\mathrm{CBD} p=.03$ ). Other patient group comparisons were non-significant. There was no overall performance difference between Italian and British or between male and female participants.

\subsection{Odour Naming}

A one-way ANOVA revealed a highly significant effect of group $(F(4,55)=71.2, p<.0001)$. Post hoc comparisons showed that each of the patient groups performed significantly worse than controls (AD versus controls $p<.0001$; FTD versus 
Table 2

Patients' performance on the Odour Perception and Semantics Battery

\begin{tabular}{|c|c|c|c|c|c|}
\hline & $\mathrm{AD}$ & FTD & SD & CBD & Controls \\
\hline Odour Discrimination/16 & $11^{* * *}(2)$ & $14(2)$ & $14(1)$ & $14(1)$ & $14(3)$ \\
\hline Odour Naming/16 & $1^{* * *}(1)$ & $3^{* * * *}(2)$ & $\mathbf{0}^{* * *}(\mathbf{0})$ & $5^{* * *}(2)$ & $10(2)$ \\
\hline Odour-to-Picture Matching/16 & $6^{* * *}(3)$ & $9^{* * *}(2)$ & $6^{* * *}(3)$ & $\mathbf{9}^{* * *}(\mathbf{3})$ & $15(1)$ \\
\hline Picture Naming/16 & $14(1)$ & $13^{*}(3)$ & $3^{* * *}(4)$ & $14(1)$ & $15(1)$ \\
\hline Word-to-Picture Matching/16 & $15(2)$ & $15(2)$ & $\mathbf{9}^{* * *}(\mathbf{3})$ & $15(1)$ & $16(0)$ \\
\hline
\end{tabular}

${ }^{*} p<.05 ;{ }^{* *} p<.01 ;{ }^{* * *} p<.001$. Comparisons are all relative to control scores. Impaired performance is shown in bold.

controls $p<.0001$; SD versus controls $p<.0001$; CBD versus controls $p<.0001)$. SD patients achieved the lowest naming scores, performing significantly worse than FTD patients $(p=.004)$ and CBD patients $(p<.0001)$. The SD versus AD comparison did not reach significance $(p=.5)$. AD patients performed more poorly than CBD patients $(p=.002)$. FTD and CBD scores did not differ significantly $(p=.51)$. There was no performance difference between Italian and British or between male and female participants.

\subsection{Odour-Picture Matching}

A one-way ANOVA revealed a highly significant effect of group $(F(4,55)=41.9, p<.0001)$. Post hoc comparisons showed that each of the patient groups performed significantly worse than controls (AD versus controls $p<.001$; FTD versus controls $p<.001$; SD versus controls $p<.001$; CBD versus controls $p<.001)$. Both $\mathrm{AD}$ and SD patients performed more poorly than CBD patients ( $p=.034$ and .026 , respectively). Other group comparisons were not significant.

Errors in the control group were minimal and therefore were not subjected to further analysis. In the case of the patient groups a two-way ANOVA (group $\times$ edibility) showed a small effect of edibility $(F(1,35)=5.2, p=.03)$. Non-edible items were more likely to elicit incorrect matching responses than edible items. There was a non-significant trend towards a group $\times$ edibility interaction effect $(F(3,35)=2.56, p=.07)$ : the poorer performance for non-edible items was highly significant in the AD group $(p=.003)$, reached significance in the SD group $(p=.02)$ but was non-significant in the FTD and CBD groups.

Distractor items in the forced-choice test were associates either of edible or inedible odours. The patient groups did not differ in the proportion of incorrect responses that were associates of inedible compared to edible items $(F(3,38)=1.14$, $p=.35)$. Moreover, the distribution of errors across the distractor alternatives did not differ significantly from chance, suggesting that patients did not have partial knowledge about unrecognised odours. There was no overall performance difference between Italian and British or between male and female participants.

\subsection{Picture Naming}

A one-way ANOVA revealed a highly significant effect of group $(F(4,55), p<.0001)$. Post hoc comparisons showed that only the SD group performed more poorly than controls $(p<.0001)$. The SD group also performed worse than AD $(p<.001), \operatorname{FTD}(p<.001)$, and $\operatorname{CBD}(p<.001)$ patients. No other group comparisons were significant. There was no significant difference in performance in Italian and British or male and female participants.

\subsection{Word-Picture Matching}

A one-way ANOVA revealed a significant effect of group $(F(4,55), p<.0001)$. Post hoc comparisons showed that only the SD group performed worse than controls $(p<.0001)$. SD patients also performed worse than $\operatorname{AD}(p<.001)$, FTD $(p<.001)$, and CBD $(p<0.001)$ patients. No other comparisons were significant. Errors were minimal in all but the SD group. In SD patients inedible items elicited more incorrect responses than edible items $\left(t_{(15)}=3.30, p=.01\right)$. The distribution of errors across the distractor alternatives did not differ significantly from chance, suggesting that patients did not have partial knowledge of unrecognised odour names. There was no significant difference in performance between Italian and British or between male and female participants.

\subsection{Inter-correlations between performance on odour tests}

In the $\mathrm{AD}$ group Odour Naming was significantly correlated with Odour-Picture Matching $\left(\rho_{(14)}=0.64, p=.01\right)$. Other correlations were non-significant. In the SD group floor level scores on Odour Naming precluded meaningful correlations. Picture Naming and Word-Picture Matching were significantly correlated $\left(\rho_{(8)}=0.76, p=.03\right)$. There was also a relationship between Odour-Picture Matching and Picture Naming, which approached significance $\left(\rho_{(8)}=0.64, p=.09\right)$. In the FTD group Odour Naming was significantly correlated with Odour-Picture Matching $\left(\rho_{(11)}=0.85, p=.001\right)$ and, in turn, performance on these two tasks correlated with their basic odour discrimination scores (with Odour Naming: $\rho_{(11)}=0.75, p=.01$; with Odour-Picture Matching: $\left.\rho_{(11)}=0.78, p=.005\right)$. The other correlations were non-significant. In the CBD group no correlations were significant.

\subsection{Correlations between performance on the odour tests and background neuropsychology}

Few significant correlations were demonstrated between performance on odour tests and background neuropsychological tasks. A small inverse relationship was demonstrated in the AD group between odour naming and animal naming on the verbal fluency test $\left(\rho_{(14)}=-0.57, p=.05\right)$ and in the SD group between odour perception and scores on the position discrimination sub- 
test of the VOSP battery $\left(\rho_{(8)}=-0.85, p=.01\right)$. In the FTD group positive relationships were demonstrated between odour matching performance and performance on the Brixton $\left(\rho_{(11)}=0.54\right.$, $p=.10)$ and Weigls $\left(\rho_{(11)}=0.70, p=.02\right)$ tests of executive function. No significant correlations were present in the CBD group.

\section{Discussion}

The study investigated the prediction that different patterns of olfactory impairment would be elicited in four forms of neurodegenerative disease. In keeping with prediction $\mathrm{AD}$ patients were impaired in the discrimination as well as the identification of odours. By contrast, SD patients were impaired in their ability to identify odours, despite normal perceptual discrimination. Performance in FTD and CBD was compromised to a much milder degree.

\subsection{Olfaction in $S D$}

SD patients show a striking dissociation between normal odour perception and severely impaired odour identification. The additional presence of impairments in picture naming and word-picture matching point to a semantic disorder as the basis for patients' impairment and is in keeping with claims that the temporal lobes have a critical role in olfactory memory (Dade, Zatorre, \& Jones-Gotman, 2002).

Published work on SD has tended to focus on word meaning. Less attention has been devoted to the exploration of other modalities (Lambon Ralph \& Howard, 2000). Nevertheless there is documented evidence that SD patients are impaired in object use (Bozeat, Lambon Ralph, Patterson, \& Hodges, 2002; Buxbaum, Schwartz, \& Carew, 1997; Hodges, Bozeat, Lambon Ralph, Patterson, \& Spatt, 2000), identification of nonverbal environmental sounds (Bozeat, Lambon Ralph et al., 2000), identification of faces (Gainotti, Barbier, \& Marra, 2003; Snowden et al., 2004) and voices (Gainotti et al., 2003), and identification from touch (Coccia et al., 2004). The findings of impairment in odour identification provide further evidence that SD patients' semantic disorder is multimodal.

In six of the SD patients there was documented evidence of altered eating habits. All six had acquired food fads. One had attempted to eat inedible objects. It would be reasonable to conclude that loss of olfactory knowledge as well as loss of visual knowledge contributes to these dietary changes in SD. SD patients also have orbitofrontal damage (Mummery, Patterson, Wise, Price, \& Hodges, 1999; Rosen et al., 2002) and, as such, we might have expected to find impaired odour discrimination in this group. We cannot rule out subtle deficits in this aspect of olfaction due to ceiling effects in control performance on the discrimination task. However, the contrast with their poor semantic performance on the smell battery indicates that: (a) semantic memory impairment is the core underlying reason for their impaired smell recognition and (b) that this is consistent with the multimodal semantic impairment observed in all other domains (Bozeat, Gregory et al., 2000; Coccia et al., 2004; Snowden et al., 1996, 2004).

\subsection{Olfaction in FTD}

FTD patients showed no impairment on our simple assessment of odour discrimination. By contrast, they were impaired in odour naming and odour-picture matching, albeit to a much lesser extent than in SD patients. Involvement of the frontal lobes in odour processing is well documented. Data from normal functional neuroimaging studies have provided evidence for the involvement of the frontal lobes in odour processing (Royet et al., 1999; Zald \& Pardo, 2000). Nevertheless, the precise basis for this involvement is open to question. Some authors have argued that the orbitofrontal cortex is important in olfactory discrimination (Zatorre \& Jones-Gotman, 1991) and we did find a correlation between odour discrimination and identification. There is some evidence from both primates (Rolls, 2001) and humans (O'Doherty et al., 2000) that the orbitofrontal areas are implicated in the judgement of pleasantness of odour and taste and in the feeling of satiety, involved in eating behaviours. Although judgement of pleasantness was beyond the scope of the present study it might be speculated that impaired judgement might contribute to the indiscriminate eating (as opposed to food fads) that are characteristic of FTD (Bathgate et al., 2001; Bozeat, Gregory et al., 2000; Ikeda et al., 2002; Miller et al., 1995; Snowden et al., 1996, 2001) and reported in 9/11 patients of the present series.

Although the presence of mild semantic impairments might contribute to FTD odour identification impairments, in view of the involvement of temporal neocortex, it is possible that there are other contributory factors. The olfactory system is capable of representing and discriminating many different odours economically, but is weak in analytic power. Thus, in humans, discrimination of odours is good whereas attribution of specific verbal labels is poor (Gloor, 1997). As identification of odours is more difficult than identification of pictures or words, it requires more active, executive control. In line with this proposal, we found sizable correlations between the FTD patients' odour identification performance and more general measures of executive function.

\subsection{Olfaction in $A D$}

The findings in $\mathrm{AD}$ are consistent with those of others, who have reported deficits in perceptual discrimination (see Liberini \& Parola, 2001; Martzke et al., 1997; Mesholam et al., 1998 for review). Comprehensive background neuropsychological data have not always been provided. However, MMSE scores (Lehrner et al., 1997; McCaffrey et al., 2000; McShane et al., 2001; Royet et al., 2001; Schiffman et al., 1990) suggest that these studies covered a wide range of disease severity. In the present study, it is notable that the disorder of odour perception occurred in the absence of detectable perceptual deficits in the visual domain. Moreover, patients' deficits were largely confined to memory and MMSE scores fell within the range of 22-26, indicating relatively mild disease. The findings emphasise that the olfactory disorder in AD is not merely a corollary of widespread cognitive impairment and advanced disease, but represents an early feature. This is consistent 
with the finding of early pathological changes not only in the hippocampal formation (Hyman, Van Hoesen, Damasio, \& Barnes, 1984), but also in piriform (Reyes, Deems, \& Suarez, 1993; Reyes et al., 1987) and entorhinal cortex (Kahn, Anderson, Miller, Wood, \& Esiri, 1987). This supports the view that olfactory deficits are an early feature of AD (Kesslak et al., 1988; Knupfer \& Spiegel, 1986; Koss et al., 1988; McCaffrey et al., 2000; Morgan et al., 1995; Rezek, 1987) and olfactory tests might contributory to early diagnosis.

Inevitably, perceptual deficits would have a secondary impact on odour identification (Eslinger, Damasio, \& Van Hoesen, 1982), so are likely to contribute to the AD patients' poor odour identification scores. Although an additional identification impairment cannot be excluded, it is noteworthy that AD patients performed normally on a verbal-visual matching test, indicating an absence of severe or generalised semantic disorder. At the same time, it has been demonstrated that AD also causes orbitofrontal damage (Van Hoesen et al., 2000) and medial orbitofrontal cortex receives inputs from the piriform cortex. As noted above, numerous lesion and functional neuroimaging studies demonstrate the importance of this region in olfaction, including olfactory discrimination. A potential role of this region cannot than ruled out, therefore.

\subsection{Olfaction in $C B D$}

The relatively mild problems of odour identification in CBD would support this notion of an executive component, since CBD does not involve the regions of the brain typically associated with olfaction. Nevertheless, CBD involves frontostriatal circuits (Lang et al., 1994) and patients are mildly impaired on executive tasks.

\section{Conclusions}

The study highlights the distinction between perceptual and semantic levels of impairment in olfaction, associated

Odour-Picture Matching task respectively with disorders that involve pyriform cortex (AD) and temporal cortex/amygdala (SD). The data from FTD and CBD data raise the possibility that executive impairments might also contribute to impaired performance on olfactory tests.

Recognition of olfactory impairment is important, not only from a theoretical perspective, but also clinically. Impaired odour perception and identification potentially exposes patients to environmental hazards such as fire and gas and increases patients' risk of ingesting stale, inedible or poisonous substances. Olfaction should not longer be the neglected sense.

\section{Acknowledgements}

We are grateful to all the participants that contributed to this study. SL was supported by a Wellcome Trust Short-term Travel Grant to MALR (ref. 068294).

\section{Appendix A}

Odour Discrimination task

\begin{tabular}{|c|c|c|c|c|}
\hline \multirow[b]{2}{*}{1} & \multicolumn{2}{|c|}{ English version } & \multicolumn{2}{|c|}{ Italian version } \\
\hline & Bleach & After-shave & Bleach & After-shave \\
\hline 2 & Coffee & Cocoa & Coffee & Cocoa \\
\hline 3 & Mint & Mint & Rosemary & Rosemary \\
\hline 4 & Petrol & Petrol & Petrol & Petrol \\
\hline 5 & Almond & Coffee & Almond & Vinegar \\
\hline 6 & After-shave & Shoe polish & After-shave & Shoe polish \\
\hline 7 & Lemon & Lemon & Lemon & Lemon \\
\hline 8 & Dettol & Dettol & Alcohol & Alcohol \\
\hline 9 & Vic & Vic & Levander & Levander \\
\hline 10 & Cocoa & Almond & Cocoa & Almond \\
\hline 11 & Shoe polish & Bleach & Shoe polish & Bleach \\
\hline 12 & Paint & Paint & Paint & Paint \\
\hline 13 & Vinegar & Whisky & Vinegar & Onion \\
\hline 14 & Orange & Orange & Orange & Orange \\
\hline 15 & Curry & Curry & Pepper & Pepper \\
\hline 16 & Whisky & Vinegar & Onion & Vinegar \\
\hline
\end{tabular}

\begin{tabular}{|c|c|c|c|c|c|}
\hline & Odour & \multicolumn{4}{|l|}{ Picture alternatives } \\
\hline \multicolumn{6}{|c|}{ (a) English version } \\
\hline 2 & Coffee & Razor & "Polo" mints & Trunk & Cup of coffee \\
\hline 3 & Mint & "Polo" mints & Chest of drawers & Cup of coffee & Cotton wool \\
\hline 4 & Petrol & Pasta & Car & Orange & Wall \\
\hline 7 & Lemon & Chocolates & Wall & Ski boot & Lemonade \\
\hline 8 & Dettol & Tool box & Orange & Cotton wool & Salad \\
\hline 9 & Vic & Nose & Cake & Razor & Garlic \\
\hline 10 & Cocoa & Car & Iron & Chocolates & Indian food \\
\hline 11 & Shoe polish & Scotsman drinking & Apple pie & Nose & Shoes \\
\hline 12 & Paint & Icecream & Lemonade & Wall & Nose \\
\hline
\end{tabular}




\begin{tabular}{rllll}
\hline & Odour & Picture alternatives & & 3 \\
\cline { 3 - 4 } & & 1 & 2 & Toilet \\
\hline 16 & & Table & Scotsman drinking & Salad \\
(b) Italian version & Whisky & & & Trunk \\
1 & Bleach & Lemonade & Envelope & Cup of coffee \\
2 & Coffee & Razor & Chest of drawers & Toilet \\
3 & Rosemary & Roast potatoes & Car & Cup of coffee \\
4 & Petrol & Pasta & Shoes & Grapes \\
5 & Almond & Cake & Razor & Cup of coffee \\
6 & After-shave & Icecream & Wall & Ski boot \\
7 & Lemon & Chocolates & Orange & Cotton wool \\
8 & Alcohol & Tool box & Cake & Razor \\
9 & Lavender & Chest of drawers & Iron & Chocolates \\
10 & Cocoa & Car & Roast chicken & Chocolates \\
11 & Shoe polish & Chest of drawers & Lemonade & Wall \\
12 & Paint & Icecream & Salad & Salad \\
13 & Vinegar & Shoes & Toilet & Garlic \\
14 & Orange & Orange juice & Cotton wool & Roast potatoes \\
15 & Pepper & Coffee & Pasta & Shoes \\
16 & Onion & Table & Roast chicken \\
\end{tabular}

Correct responses are in bold. 1-4 correspond to locations on the page: 1 upper left; 2 upper right; 3 bottom left; 4 bottom right.

\section{References}

Bathgate, D., Snowden, J. S., Varma, A., Blackshaw, A., \& Neary, D. (2001). Behaviour in frontotemporal dementia Alzheimer's disease and vascular dementia. Acta Neurologica Scandinavica, 103, 367-378.

Bozeat, S., Gregory, C. A., Lambon Ralph, M. A., \& Hodges, J. R. (2000). Which neuropsychiatric and behavioural features best distinguish frontal and temporal variants of frontotemporal dementia from Alzheimer's disease. Journal of Neurology Neurosurgery \& Psychiatry, 6, 178186.

Bozeat, S., Lambon Ralph, M. A., Patterson, K., Garrard, P., \& Hodges, J. R. (2000). The nonverbal semantic impairment in semantic dementia. Neuropsychologia, 38, 1207-1215.

Bozeat, S., Lambon Ralph, M. A., Patterson, K., \& Hodges, J. R. (2002). When objects lose their meaning: What happens to their use? Cognitive, Affective \& Behavioral Neuroscience, 2(3), 236-251.

Brandt, J. (1991). The Hopkins Verbal Learning Test: Developmental of a new memory test with six equivalent forms. The Clinical Neuropsychologist, 5 , $125-142$.

Burgess, P. W., \& Shallice, T. (1997). The Haylings and Brixton test. Bury St Edmunds, England: Thames Valley Test Company.

Buxbaum, L. J., Schwartz, M. F., \& Carew, T. G. (1997). The role of semantic memory in object use. Cognitive Neuropsycology, 14, 219254.

Caltagirone, C., Gainotti, G., Masullo, C., \& Miceli, G. (1979). Validity of some neuropsychological tests in the assessment of mental deterioration. Acta Psychiatrica Scandinavica, 60, 50-56.

Coccia, M., Bartolini, M., Luzzi, S., Provinciali, L., \& Lambon Ralph, M. A. (2004). Semantic memory is an amodal, dynamic system: Evidence from the interaction of naming and object use in semantic dementia. Cognitive Neuropsycology, 21(5), 513-527.

Dade, L. A., Zatorre, R. J., \& Jones-Gotman, M. (2002). Olfactory learning: Convergent findings from lesion and brain imaging studies in humans. Brain, $125,86-101$

Di Nuovo, S. (1979). Il test di Rey: figura complessa B. Firenze: Organizzazioni Speciali S.r.l.

Doty, R. L., Reyes, P. F., \& Gregor, T. (1987). Presence of both odor identification and detection deficits in Alzheimer's disease. Brain Research Bulletin, 18, 597-600.

Doty, R. L., Shaman, P., \& Dann, M. (1984). Development of the University of Pensylvania Odour Identification Test: A standardized microencapsulated test of olfactory function. Physiology \& Behavior, 32, 489-502.
Eslinger, P. J., Damasio, A. R., \& Van Hoesen, G. W. (1982). Olfactory dysfunction in man: Anatomical and behavioural aspects. Brain \& Cognition, 1, 259-285.

Folstein, M. F., Folstein, J. E., \& McHugh, P. R. (1975). Mini-Mental State. Journal of Psychiatric Research, 12, 189-198.

Gainotti, G., Barbier, A., \& Marra, C. (2003). Slowly progressive defect in recognition of familiar people in a patient with right anterior temporal atrophy. Brain, 126, 792-803.

Gloor, P. (1997). The temporal lobe and the limbic system. Oxford: Oxford University Press.

Gustafson, L., Brun, A., \& Risberg, J. (1987). Frontal lobe degeneration of nonAlzheimer type II. Clinical picture and differential diagnosis. Archives of Gerontology and Geriatrics, 6(3), 209-223.

Hawkes, C. (2003). Olfaction in neurodegenerative disorder. Movement Disorders, 18, 364-372.

Hodges, J. R., Bozeat, S., Lambon Ralph, M. A., Patterson, K., \& Spatt, J. (2000). The role of conceptual knowledge in object use: Evidence from semantic dementia. Brain, 123, 1913-1925.

Hyman, B. T., Van Hoesen, G. W., Damasio, A. R., \& Barnes, C. L. (1984) Alzheimer's disease: Cell-specific pathology isolates the hippocampal formation. Science, 225, 1168-1170.

Ikeda, M., Brown, J., Holland, A. J., Fukuhara, R., \& Hodges, J. R. (2002). Changes in appetite, food preference and eating habits in frontotemporal dementia and Alzheimer's disease. Journal of Neurology Neurosurgery \& Psychiatry, 73, 371-376.

Kahn, J., Anderson, B. H., Miller, C. C., Wood, J. N., \& Esiri, M. M. (1987). Staining with monoclonal antibodies to neurofilaments distinguishes between subpopulations of neurofibrillary tangles, between groups of axons and between groups of dendrites. Journal of Neurology, 234, 241 246.

Kesslak, J. P., Cotman, C. W., Chui, H. C., Van Der Noort, S., Fang, H., Pfeffer, R., et al. (1988). Olfactory tests as possible probes for detecting and monitoring Alzheimer's disease. Neurobiology of Aging, 9, 399-403.

Knupfer, L., \& Spiegel, R. (1986). Differences in olfactory test performance between normal and aged Alzheimer and vascular type dementia individuals. International Journal of Geriatric Psychiatry, 1, 3-14.

Koss, E., Weiffenbach, J. M., Haxby, J. V., \& Friedland, R. P. (1988). Olfactory detection and identification performance are dissociated in early Alzheimer's disease. Neurology, 38, 1228-1232.

Lambon Ralph, M. A., \& Howard, D. (2000). Gogi aphasia or semantic dementia? Assessing poor verbal comprehension in a case of fluent progressive aphasia. Cognitive Neuropsychology, 17, 437-466. 
Lang, A. E., Riley, D. E., \& Bergeron, C. (1994). Cortical-basal ganglionic degeneration. In D. B. Calne (Ed.), Neurodegenerative diseases (pp. 877-894). Philadelphia: W.B. Saunders.

Larsson, M., Semb, H., Winblad, B., Amberla, K., Wahlund, L. O., \& Bäckman, L. (1999). Odor identification in normal aging and early Alzheimer's disease: Effects of retrieval support. Neuropsychology, 13(1), 47-53.

Lehrner, J. P., Brücke, T., Dal-Bianco, P., Gatterer, G., \& Kryspin-Exner, I. (1997). Olfactory functions in Parkinson's disease and Alzheimer's disease. Chemical Senses, 22, 105-110.

Liberini, P., \& Parola, S. (2001). Olfaction in neurodegenerative diseases. In F. Boller \& S. F. Cappa (Eds.), Handbook of neuropsychology, Vol. 6, 2nd ed. (pp. 145-161). Amsterdam: Elsevier Science.

Martzke, J. S., Kopala, L. C., \& Good, K. P. (1997). Olfactory dysfunction in neuropsychiatric disorders: Review and methodological considerations. Biological Psychiatry, 42, 721-732.

McCaffrey, R. J., Duff, K., \& Solomon, G. S. (2000). Olfactory dysfunction discriminates probable Alzheimer's dementia from major depression: A cross-validation and extension. The Journal of Neuropsychiatry and Clinical Neurosciences, 12, 29-33.

McKhann, G., Drachman, D., Folstein, M., Katzman, R., Price, D., \& Stranlan, E. M. (1984). Clinical diagnosis of Alzheimer's disease: Report of the NINCDS-ADRDA work group under the auspices of Department of Health and Human Services Task Force on Alzheimer's Disease. Neurology, 34, 939-944.

McShane, R. H., Nagy, Z., Esiri, M. M., King, E., Joachim, C., Sullivan, N., et al. (2001). Anosmia in dementia is associated with Lewy bodies rather than Alzheimer's pathology. Journal of Neurology Neurosurgery \& Psychiatry, 70, 739-743.

Mesholam, R. I., Moberg, P. J., Mahr, R. N., \& Doty, R. L. (1998). Olfaction in neurodegenerative disease. A meta-analysis of olfactory functioning in Alzheimer's and Parkinson's diseases. Archives of Neurology, 5, 84-90.

Miller, B. L., Darby, A. L., Swartz, J. R., Yener, G. G., \& Mena, I. (1995). Dietary changes, compulsions and sexual behavior in frontotemporal degeneration. Dementia, 6, 195-199.

Morgan, C. D., Nordin, S., \& Murphy, C. (1995). Odor identification as an early marker for Alzheimer's disease: Impact of lexical functioning and detection sensitivity. Journal of Clinical and Experimental Neuropsychology, 17(5), 793-803.

Müller, A., Reichmann, H., Livermore, A., \& Hummel, T. (2002). Olfactory function in idiopathic Parkinson's disease (IPD): Results from crosssectional studies in IPD patients and long-term follow-up of de novo IPD patients. Journal of Neural Transmission, 109, 805-811.

Mummery, C. J., Patterson, K., Wise, R. J. S., Price, C. J., \& Hodges, J. R. (1999). Disrupted temporal lobe connections in semantic dementia. Brain, $122,61-73$

Nauta, W. J. H., \& Feirtag, M. (1986). Fundamental neuroanatomy. New York: W.H. Freeman \& Co.

Neary, D., Snowden, J. S., Gustafson, L., Passant, U., Stuss, D., Black, S., et al. (1998). Frontotemporal lobar degeneration. Neurology, 51, 1546-1554.

O’Doherty, J., Rolls, E. T., Francis, S., Bowtell, R., McGlone, F., Kobal, G., et al. (2000). Sensory-specific satiety related olfactory activation of the human orbitofrontal cortex. NeuroReport, 11, 399-402.
Parola, S., \& Liberini, P. (1999). Assessing olfaction in the Italian population: Methodology and clinical application. Italian Journal of Neurological Sciences, 20, 287-296.

Reyes, P. F., Deems, D. A., \& Suarez, M. G. (1993). Olfactory-related changes in Alzheimer's disease: A quantitative neuropathologic study. Brain Research Bulletin, 32, 1-5.

Reyes, P. F., Golden, G. T., Fagel, P. L., Fariello, R. G., Katz, L., \& Carner, E. (1987). The prepiriform cortex in dementia of the Alzheimer type. Archives of Neurology, 44, 644-645.

Rezek, D. (1987). Olfactory deficits as a neurological sign in dementia of Alzheimer type. Archives of Neurology, 44, 1030-1032.

Rolls, E. T. (2001). The rules of formation of the olfactory representations found in the orbitofrontal cortex olfactory areas in primates. Chemical Senses, 26, 595-604.

Rosen, H. J., Gorno-Tempini, M. L., Goldman, W. P., Perry, R. J., Schuff, N., Weiner, M., et al. (2002). Neurology, 58, 198-208.

Royet, J. P., Croisile, B., Williamson-Vasta, R., Hibert, O., Serclerat, D., \& Guerin, J. (2001). Rating of different olfactory judgements in Alzheimer's disease. Chemical Senses, 26, 409-417.

Royet, J. P., Koenig, O., Gregoire, M. C., Cinotti, L., Lavenne, F., Le Bars, D., et al. (1999). Functional anatomy of perceptual and semantic processing for odors. Journal of Cognitive Neuroscience, 11(1), 94-109.

Schiffman, S. S., Clark, C. M., \& Warwick, Z. S. (1990). Gustatory and olfactory dysfunction in dementia: Not specific to Alzheimer's disease. Neurobiology of Aging, 11, 597-600.

Serby, M., Larson, P., \& Kalkstein, D. (1991). The nature and course of olfactory deficits in Alzheimer's disease. The American Journal of Psychiatry, 148, 357-360.

Snowden, J. S., Bathgate, D., Varma, A., Blackshaw, A., Gibbons, Z. C., \& Neary, D. (2001). Distinct behavioural profiles in frontotemporal dementia and semantic dementia. Journal of Neurology Neurosurgery \& Psychiatry, $70,323-332$.

Snowden, J. S., Neary, D., \& Mann, D. M. A. (1996). Frontotemporal lobar degeneration: Frontotemporal dementia, progressive aphasia, semantic dementia. London: Churchill Livingstone.

Snowden, J. S., Thompson, J. C., \& Neary, D. (2004). Knowledge of famous faces and names in semantic dementia. Brain, 127, 860872

Spinnler, H., \& Tognoni, G. (1987). Standardizzazione etaratura italiana di test neuropsicologici. Italian Journal of Neurological Sciences, (Suppl. 8).

Van Hoesen, G. H., Parvizi, J., \& Chu, C. C. (2000). Orbitofrontal cortex pathology in Alzheimer's disease. Cerebral Cortex, 10(3), 243-251.

Warrington, E. K., \& James, M. (1991). The visual object and space perception battery. Bury St Edmunds: Thames Valley Test Company.

Wenning, G. K., Shephard, B., Hawkes, C., Petruckevitch, A., Lees, A., \& Quinn, N. (1995). Olfactory function in atypical parkinsonian syndromes. Acta Neurologica Scandinavica, 91, 247-250.

Zald, D. H., \& Pardo, J. V. (2000). Functional neuroimaging of the olfactory system in humans. International Journal of Psychophysiology, 36, 165181

Zatorre, R., \& Jones-Gotman, M. (1991). Human olfactory discrimination after unilateral frontal or temporal lobectomy. Brain, 114, 71-84. 